\title{
Effect of combined application of Trichoderma harzianum and Bacillus subtilis against wilt disease complex of chickpea caused by Fusarium oxysporum and Rhizoctonia solani
}

MANISH KUMAR JAIN*, SIMMI JAIN AND S. BANERJEE

Department of Biotechnology, Dr. H.S. Gour University, SAGAR (M.P.) INDIA

\section{ARITCLE INFO \\ Received : 27.05 .2014 \\ Revised : 07.01 .2015 \\ Accepted : 21.01 .2015}

\section{KEY WORDS :}

Chickpea, Bacillus subtilis, Trichoderma harzianum, Fusarium oxysporium, Legume, Antagonist

*Corresponding author: Email: sunny9jan@gmail.com

\begin{abstract}
In this study, antagonistic effects of Bacillus subtilis and Trichoderma harzianum isolated from Rhizosphere of chickpea were evaluated against Fusarium oxysporum as potential biocontrol agents in vitro and in vivo. Fungal inhibition tests were performed using plate assay. Isolates were selected according to their high antagonistic efficiency in in vitro which was shown as inhibition zones in the dual-culture assay. Bacillus subtilis was isolated in cyst form and is transferred to inert carriers like peat, lignite ore or can be transferred to liquid medium. In lab conditions (controlled) seeds of chickpea are 20 treated directly with Bacillus sp. culture and dried for a while and treated with cultures (generally spores) of Trichoderma sp. These treated seeds are sown in earthern pots containing black soil having few spores of Fusarium oxysporium (MTCC, Chandigarh). Observations are made after every 2-4 days. Combined application of Bacillus subtilis and Trichoderma harzianum has synergistic effect on the growth of Fusarium oxysporium in in-vitro environment. Our results indicate that PGPR improve growth parameters in this plant and can help in the biocontrol of pathogen.

How to view point the article : Jain, Manish Kumar, Jain, Simmi and Banerjee, S. (2015). Effect of combined application of Trichoderma harzianum and Bacillus subtilis against wilt disease complex of chickpea caused by Fusarium oxysporum and Rhizoctonia solani. Internat. J. Plant Protec., 8(1) : 21-25.
\end{abstract}

